\title{
SwissDRG: Ausschreibung für begleitende Untersuchung
}

\author{
FMH und $\mathrm{H}+$ schreiben gemeinsam eine wissenschaftliche Untersuchung zu den Leis- \\ tungs- und Kostenverschiebungen zwischen dem stationären und dem ambulanten \\ Sektor aus.
}

Beatrix Meyer

Leiterin Tarifdienst FMH und Bereich SwissDRG

Seit mehreren Jahren weist die FMH auf die Wichtigkeit der Begleitforschung aus Anlass der Einführung von SwissDRG hin. An konkreten Empfehlungen hat es dabei nicht gefehlt: Bereits 2009 reichte die FMH ein Konzept für die Umsetzung der Begleitforschung bei der SwissDRG AG ein [1]. Eine der Empfehlungen des FMH-Konzepts war, die Leistungs- und Kostenverschiebungen zwischen dem stationären und spital-/praxisambulanten Sektor zu untersuchen. Doch leider hatte die SwissDRG AG entschieden, nicht Hauptträgerin der Begleitforschung zu werden, so dass die Empfehlungen des FMH-Konzepts nicht umgesetzt wurden.

\section{Die Studie soll nicht nur das Ausmass der Verlagerung, sondern auch die Gründe dafür untersuchen.}

Korrespondenz: Beatrix Meyer Tarifdienst FMH Froburgstrasse 15 CH-4600 Olten Tel. 0313591111

swissdrg@fmh.ch
Nach dieser Entscheidung der SwissDRG AG hoffte die Ärzteschaft, dass sich das Bundesamt für Gesundheit (BAG) der Thematik annimmt. In der Machbarkeitsstudie des BAG zur Evaluation der KVG-Revision wurde dann zwar in einem der 15 Teilprojekte vorgeschlagen, die Kostenverschiebungen vom stationären in den ambulanten Sektor zu untersuchen [2]. Doch der Umsetzungsentscheid des BAG ist seit Mitte 2010 ausstehend. Und selbst wenn sich das BAG doch noch dazu entscheidet, diesen Aspekt untersuchen zu lassen, wird das vorgesehene Vorgehen den Bedürfnissen der Leistungserbringer nicht gerecht. Denn die Autoren der BAG-Machbarkeitsstudie empfehlen, mit dem entsprechenden Teilprojekt erst im Jahr 2013 zu starten. Und die BAG-Machbarkeitsstudie regt eine Sekundäranalyse an, obwohl dazu im spitalambulanten Sektor die entsprechenden Daten fehlen.

Wer kümmert sich denn nun um das für die Ärzteschaft zentrale Thema der Kosten- und Leistungsverschiebungen zwischen dem akutstationären und dem spital-/praxisambulanten Sektor? Ist zu befürchten, dass wir wie unsere deutschen Kollegen vergeblich auf entsprechende Untersuchungen hoffen? Um dies zu vermeiden, hat die FMH das Gespräch mit H+ aufgenommen. Beide Akteure haben beschlossen, eine gemeinsame Ausschreibung zu lancieren und die Thema- tik wissenschaftlich untersuchen zu lassen. Interessenten finden die Ausschreibung auf www.fmh.ch $\rightarrow$ Tarife $\rightarrow$ SwissDRG $\rightarrow$ Begleitforschung. So kann die Untersuchung zwar erst spät, aber doch noch vor der Einführung von SwissDRG starten.

Die Studie soll nicht nur das Ausmass der allfälligen Verlagerung von Abklärungen und Behandlungen vom akutstationären in den spital-/praxisambulanten Sektor, sondern auch die Gründe dafür untersuchen. Welchen Einfluss hat dabei DRG? Welchen Einfluss hat z. B. die Entwicklung der Tageschirurgie? Dank dieser Studie sollen die Leistungserbringer in Zukunft über verlässliche Daten verfügen und gegenüber Politikern und Partnern mit Zahlen und Fakten argumentieren können. Zudem sollen allfällige Verkürzungen der Liegezeiten im Spital und Verlagerungen von Untersuchungen und Behandlungen in den ambulanten Sektor nicht fälschlicherweise als «Mengenausweitung» identifiziert werden.

Allerdings wird mit dieser Untersuchung sowie mit der von der FMH lancierten Studie zur Entwicklung der Rahmenbedingungen der Spitalärzte und der Arzt-Patienten-Beziehung [3] nur ein Bruchteil der von der FMH geforderten Begleitforschung umgesetzt [1]. Die FMH und $\mathrm{H}+$ müssen sich hier auf die für ihre Mitglieder wichtigsten Themen konzentrieren. Damit das Ziel eines umfassenden Monitorings als Grundlage für eine nachhaltige Gesundheitspolitik erreicht wird, sind gemeinsame Anstrengungen aller Partner im Gesundheitswesen notwendig.

\section{Literatur}

1 Bovier P, Burnand B, Guillain H, Paccaud F, Vader JP, Locher H, Meyer B. Konzept für eine Begleitforschung aus Anlass der Einführung von SwissDRG, www.fmh.ch $\rightarrow$ Tarife $\rightarrow$ SwissDRG $\rightarrow$ Begleitforschung; 2009.

2 Pellegrini S, Widmer T, Weaver F, Fritschi T, Bennett J. KVG-Revision Spitalfinanzierung: Machbarkeits- und Konzeptstudie zur Evaluation. Schlussbericht, im Auftrag des Bundesamtes für Gesundheit. www.bag.admin.ch/evaluation/ 01759/07350/07641/index.html?lang=de; 2010, S. 82f.

3 Begleitende Untersuchung aus Anlass der Einführung von SwissDRG: Entwicklung der Rahmenbedingungen für die Spitalärzte und der Arzt-Patienten-Beziehung. vgl. www.fmh.ch $\rightarrow$ Tarife $\rightarrow$ SwissDRG $\rightarrow$ Begleitforschung. 